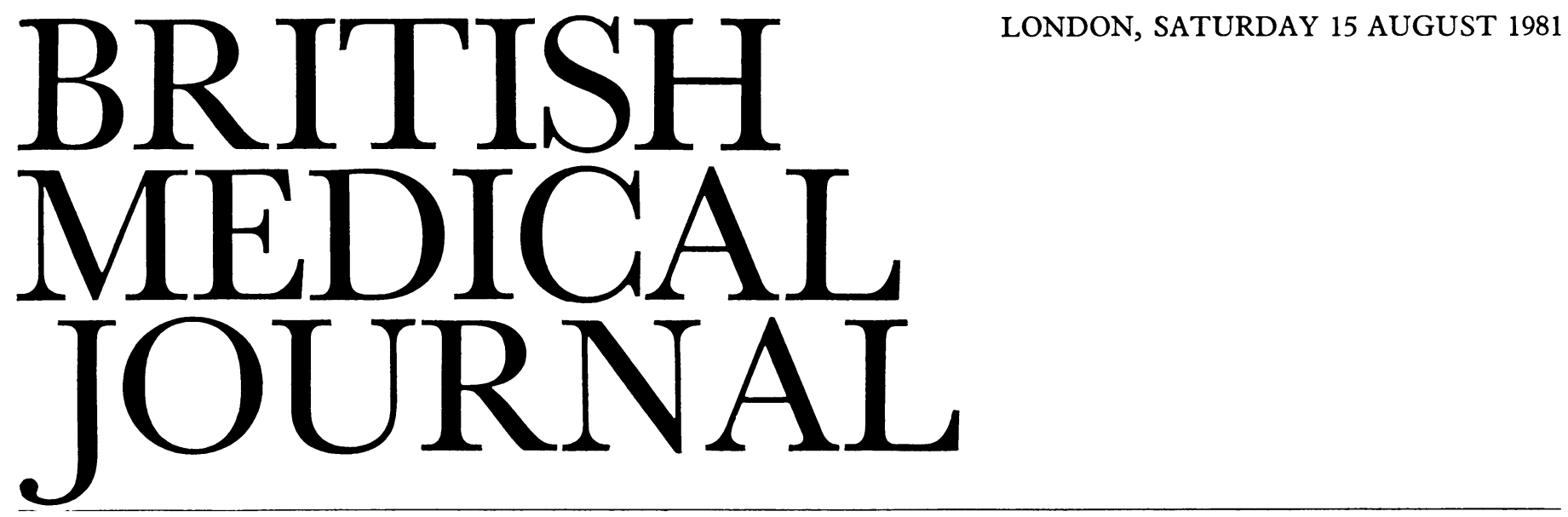

The association between severe alcoholism and brain damage dates back to the classic report in 1881 by Wernicke of three patients with acute tremor, ataxia, ophthalmoplegia, and delirium who were found at necropsy to have numerous petechial haemorrhages around the third and fourth ventricles; two of the three were alcoholics. Six years later Korsakoff described the characteristic amnesia with confabulation, but neither Korsakoff nor Wernicke appreciated the link between their eponymous conditions. ${ }^{1}$ A further half-century was to pass before it was shown that thiamine could dramatically improve Wernicke's encephalopathy and could also ameliorate some cases of Korsakoff's psychosis. Eventually the pathological changes in the two conditions were shown to be similar, and in 1971 Victor et $a l^{2}$ convincingly showed that 155 of 186 alcoholic patients who survived the acute illness went on to develop chronic amnesia.

That is the clear-cut picture as presented to medical students, who are still taught that only a few severe alcoholics, perhaps through some accident of diet or enzymatic predisposition, ${ }^{3}$ develop the Wernicke-Korsakoff syndrome and that when they do the diagnosis is easy. Unfortunately life, and death, are rarely as simple as undergraduate textbooks suggest. Harper ${ }^{4}$ recently reported that $2^{\prime \prime}$ " of all brains examined post mortem in a Perth hospital showed Wernicke's encephalopathy; in only seven of the 51 cases of the condition had the diagnosis been suspected during life. Also in Australia, Horvath" found that 100 out of 1100 patients seen at an alcoholism clinic had a chronic brain syndrome as defined by progressive failure of memory, deterioration of personality, and loss of intellectual ability. Only 20 of these 100 patients had classical Korsakoff's defects, while the remainder suffered from a combination of failure of memory, behavioural disturbance, apathy, and, in some cases, specific neurological deficits.

Findings such as these raise two questions: Are many mild cases of Wernicke-Korsakoff's syndrome being missed? Is there an alcoholic "dementia" distinct from WernickeKorsakoff's syndrome? Thirty years ago Courville ${ }^{6}$ argued for this latter notion, maintaining that alcoholism was the commonest cause of shrinkage of the brain in the fifth and sixth decades. More recently air-encephalographic studies have also shown an excess of cerebral atrophy among alcoholics. Brewer and Perrett, ${ }^{7}$ for instance, found that frontal and parietal atrophy was common in 33 heavy drinkers, and 24 of their cases showed ventricular enlargement.
The advent of non-invasive computed tomography greatly facilitated the search for possible alcohol-induced brain lesions. Fox et al ${ }^{8}$ reported enlarged ventricles in four out of 12 alcoholics with liver disease, while Epstein et $a l^{9}$ found that 28 of their 46 alcoholics showed cerebral atrophy on computed tomography. Cala et al ${ }^{10}$ reported severe cortical atrophy in half of their 26 Australian alcoholics, while Bergman et al ${ }^{11}$ noted cortical changes in 61 of 106 consecutive patients admitted to an alcoholic unit in Stockholm. Similar findings were reported from Canada. ${ }^{12}$

These investigators did not all examine homogeneous groups of patients and their criteria for inclusion varied widely. One study which did use strict diagnostic criteria for alcoholism and also examined abstemious or moderate drinking controls was that of Ron and her colleagues ${ }^{13}$ at the Institute of Psychiatry in London. They carried out scans after a mean "drying-out" period of 34 days which were rated "blind." Any patient in whom brain damage was suspected clinically was excluded. Over 40 of the 100 clinically unimpaired alcoholic men had increased widening of the sulcus compared with only two of the 50 controls, while the mean ventricle to brain ratio was roughly half as large again in the alcoholics as in the controls. Most interestingly, significant differences emerged between alcoholics and controls below the age of 40 as well as in older subjects.

Since these CT scan abnormalities are seen in alcoholics who appear clinically normal are they of any functional importance? Routine intelligence testing provides no evidence that alcoholics as a group show overall intellectual impairment. ${ }^{14}$ Specific defects do, however, appear in tasks entailing nonverbal abstracting abilities, while deficits may also be found on complex perceptual motor tasks. ${ }^{15}$ Many attempts have been made to link such deficits with observed changes in the brain. Tarter, in a paper presented to the conference "Evaluation of the alcoholic," University of Connecticut, October 1979 , found that people with personality or mental deterioration were more likely than non-deteriorated alcoholics to show definite dilatation of the ventricles on pneumoencephalography, while Brewer and Perrett ${ }^{7}$ found a correlation of 0.48 between cortical atrophy and the results of the Benton Visual Retention Test. Correlations between neuropsychological performance and CT scan abnormalities have tended to be less definite.

Among the many suggestions about the cause of alcoholrelated brain impairment is the possibility that minimal brain 
dysfunction might be a cause rather than a consequence of alcoholism. ${ }^{16}$ Malnutrition, repeated head injury, and concomitant liver disease have also been suspected, but Eckardt et $a l^{17}$ could find no evidence in favour of these and suggested instead a direct toxic effect of alcohol. Stopping drinking certainly seems beneficial, with improvement in psychological performance within the first few weeks of abstinence. ${ }^{17}$ Improvement can also occur in the CT scan appearances, though these changes are slower and less striking. ${ }^{1}$

At present, therefore, it seems that almost one in 10 patients presenting to alcoholic units will have a clinically obvious organic brain syndrome. Shrinkage of the brain can be shown in over half of the remaining $90 \%$ who appear clinically "intact." Cognitive impairment is frequently present, with problem-solving ability, abstract thinking, psychomotor speed, and memory particularly vulnerable.

What importance do these findings have for everyday practice? Certainly clinicians should be more alert to the possibility of minor brain damage, and any alcoholic who still has even minor disorientation, loss of memory, or difficulty in learning new material after two or three weeks' abstinence deserves a full neuropsychological investigation. But are there wider implications? Could minor brain damage play a part in precipitating loss of control of drinking? Could subtle intellectual impairment rather than moral weakness explain the poor judgment and repeated relapse into drinking shown by so many alcoholics? Is the prognosis worse for those with brain shrinkage? Are some alcoholics too impaired to grasp the complicated concepts so often discussed during group therapy? Would the authoritarian approach of enforced abstinence for three months allow abstract thinking to improve sufficiently so that more alcoholics could make a balanced judgment about the pros and cons of further drinking? Researchers have shied away from these difficult clinical questions, but they should do so no longer.

1 Lishman WA. Cerebral disorder in alcoholism syndromes of impairment. Brain 1981;104:1-20.

2 Victor M, Adams RD, Collins GH. The Wernicke-Korsakoff syndrome. Oxford: Blackwell Scientific Publications, 1971.

${ }^{3}$ Blass JP, Gibson GE. Genetic factors in the Wernicke-Korsakoff syndrome. Alcoholism: Clinical and Experimental Research $1979 ; 3: 126-34$.

4 Harper C. Wernicke's encephalopathy: a more common disease than realised. A neuropathological study of 51 cases. $\mathcal{F}$ Neurol Neurosurg Psychiatry 1979;42:226-31.

${ }^{5}$ Horvath TB. Clinical spectrum and epidemiological features of alcoholic dementia. In: Rankin JD, ed. Alcohol, drugs and brain damage. Toronto Alcoholism and Drug Addiction Research Foundation, 1975;1-16.

${ }^{6}$ Courville CB. The effects of alcohol on the nervous system of man. Los Angeles: San Lucas Press, 1955.

${ }^{7}$ Brewer C, Perrett L. Brain damage due to alcohol consumption: an air-encephalographic, psychometric and electroencephalographic study. Br $\mathcal{F}$ Addict $1971 ; 66: 170-82$.

${ }^{8}$ Fox JH, Ramsey RG, Huckman MS, Proske AE. Cerebral ventricular enlargement. Chronic alcoholics examined by computerized tomography. 7AMA 1976;236:365-8.

${ }^{\circ}$ Epstein PS, Pisani VD, Fawcett JA. Alcoholism and cerebral atrophy Alcoholism: Clinical and Experimental Research 1977;1:61-5.

${ }^{10}$ Cala LA, Jones B, Mastaglia FL, Wiley B. Brain atrophy and intellectual impairment in heavy drinkers-a clinical, psychometric and computerized tomography study. Aust NZ F Med 1978;8:147-53.

11 Bergman H, Borg S, Hindmarsh T, Idestrom C-M, Mützell S. Computed tomography of the brain and neuropsychological assessment of alcoholic patients. In: Begleiter $\mathrm{H}$, ed. Biological effects of alcohol. New York: Plenum Press, 1980:771-86.

12 Wilkinson A, Carlen PL. Relation of neuropsychological test performance in alcoholics to brain morphology measured by computed tomography. In: Begleiter H, ed. Biological effects of alcohol. New York: Plenum Press, 1980:683-99.

13 Ron MA, Acker W, Lishman WA. Morphological abnormalities in the brains of chronic alcoholics. A clinical, psychological and computerized axial tomographic study. Acta Psychiatr Scand 1980;62, suppl 286:51-6.

14 Tarter RE. Brain damage in chronic alcoholics. A review of the psychological evidence. In : Richter D, ed. Addiction and brain damage. London Croom Helm, 1980:267-97.
15 Parsons OA. Neuropsychological deficits in alcoholics: facts and fancies. Alcoholism: Clinical and Experimental Research 1977;1:51-6.

16 Tarter RE. Minimal brain dysfunction as an etiological predisposition to alcoholism

17 Eckardt MJ, Parker ES, Noble EP, Feldman DJ, Gottschalk LA Relationship between neuropsychological performance and alcohol consumption in alcoholics. Biol Psychiatry 1978;13:551-65.

${ }^{18}$ Guthrie A. The first year after treatment: factors affecting time course of reversibility of memory and learning deficits in alcoholism. In: Begleiter $\mathrm{H}$, ed. Biological effects of brain damage. New York: Plenum Press, 1980:757-70.

\section{Management of gastrointestinal bleeding}

The diagnostic revolution of the last 15 years has greatly helped the surgeon who has to deal with emergency gastrointestinal bleeding. Not only do fewer patients come his way undiagnosed with requests for heroic operations such as total gastrectomy, but nowadays he seldom needs to open the stomach to examine the gastric and duodenal mucosa. Emergency endoscopy has also virtually eliminated the need for less reliable urgent barium-meal examinations and provides a positive diagnosis in about $90^{\circ}$ of patients who bleed. ${ }^{1}$ Emergency endoscopy should not, however, be undertaken indiscriminately, since most patients have stopped bleeding by the time they are admitted to hospital, and in most the bleeding does not recur. ${ }^{2}$ Several trials (albeit with weaknesses in design ${ }^{3}$ ) have failed to show any benefits from emergency endoscopy, and the latest careful study ${ }^{4}$ found no advantages in terms of management, reduction of mortality, and long-term consequences. The consensus must surely be that routine endoscopy in every patient will result in unnecessary discomfort to many-besides being a waste of time and money.

In certain subgroups of patients, ${ }^{3}$ however, endoscopy is needed to plan definitive treatment, though the procedure may be technically difficult. The most important group consists of patients who continue to bleed or in whom bleeding recurs. An emergency service should also take account of the population at risk: for example, an English series would be unlikely to contain as many as $26^{\%} \%$ of cirrhotics, ${ }^{4}$ in whom endoscopy may be particularly valuable in defining the exact site of bleeding.

If, then, accurate diagnosis does not affect the overall outcome in most cases, we must look carefully at other aspects of management ${ }^{5}$ to see if we can reduce the hard core of $5-10 \%$ of patients who die. As with all emergencies, management should be based on a system of priorities. During resuscitation attention needs to be concentrated on the cardiovascular system, with only limited concern being given to the cause of the bleeding. Once the clinical condition has been stabilised, a full history may be obtained and physical examination carried out, but this will provide a diagnosis only in perhaps two-thirds of patients. Some gastroenterologists argue that all such patients should be admitted to special wards with facilities for intensive care such as central venous pressure monitoring, an attached endoscopy suite, and combined medical and surgical cover. The danger is that each patient will then get the full treatment, when it is needed by at most $10^{\circ} \%$ whose condition does not settle. These patients are the ones in whom to consider endoscopy and other diagnostic measures. Aspiration of blood from the stomach may not only confirm that bleeding is continuing but may indicate its site. The results of selective angiography may be valuable, especially when bleed- 